all Aristolochiaceae could be responsible for this effect, and Schvartzman et al. ${ }^{15}$ reported that aristolochic acid I, which is found in almost all the species of this genus ${ }^{16}$, has c-mitotic effects.

No mitotic alterations were observed in roots treated with infusions of S. rebaudiana, as can be deduced from the figure A. The evolution of mitotic indices after $24 \mathrm{~h}$ treatment is quite normal in all cases. Nevertheless, cell cycle duration is prolonged by treatments with these infusions, as can be seen in the figure $B$, which shows the entrance in mitosis of a synchronous binucleate subpopulation in control and treated roots. Finally, cytophoto- metrical studies showed that the distribution of cells in $\mathrm{G}_{1}, \mathrm{~S}$ and $\mathrm{G}_{2}$, amounting to $26 \%, 45 \%$ and $16 \%$ respectively in the controls, was not significantly modified by these treatments. Extracts of S. rebaudiana were found to have contraceptive properties in rats ${ }^{17}$. Our experiments demonstrate that infusions of this plant have no specific toxicological effects upon the cell cycle of A. cepa meristems, suggesting that the contraceptive properties may not be connected with the chromosome cycle ${ }^{15}$.

17 G. M. Planas, Science 162, 1007 (1967).

\title{
Ventral motor neuron alterations in rat spinal cord after chronic exercise ${ }^{1,2}$
}

\author{
T. B. Gilliam, R. R. Roy ${ }^{3}$, J. F. Taylor, W. W. Heusner and W. D. Van Huss \\ Department of Physical Education, 401 Washtenaw, The University of Michigan, Ann Arbor (Michigan 48109, USA),
} 27 September 1976

Summary. The observed differences in the soma and nuclear diameters reflect chronic changes specific to each exercise regimen used.

A dearth of information exists concerning the effects of physical activity on the size of the cell body and the nucleus of the ventral motor neuron. Acute bouts of swimming or running have been reported to increase ${ }^{4,5}$, decrease $^{6-8}$, or not change ${ }^{9}$ the volume of the soma. Similar conflicting results have been found for changes in nuclear sizes $4,5,9$. An inspection of the exercise protocols used indicates that the inconsistency in these results may

Table 1 . Summary of $\chi^{2}$-values for soma and nucleus distribution differences between groups after 12 weeks of training

\begin{tabular}{llll}
\hline Comparison & & df & 12 weeks $\chi^{2}$ \\
\hline \multirow{2}{*}{ CON* vs SPT* $^{*}$} & Soma & 30 & $73.44^{* *}$ \\
& Nucleus & 14 & $77.87^{* *}$ \\
CON vs END* & Soma & 30 & $53.39^{* *}$ \\
& Nucleus & 14 & $42.83^{* *}$ \\
SPT vs END & Soma & 30 & $44.94^{* *}$ \\
& Nucleus & 14 & $39.40^{* *}$ \\
\hline
\end{tabular}

${ }^{*} \mathrm{n}, 4$ animals. ** Significant distribution differences at the 0.05 level.

Table 2. Summary of $\chi^{2}$-values for soma and nucleus distribution difference between pooled-zero** and 12-week animals for each treatment group

\begin{tabular}{llllllll}
\hline Comparison & \multicolumn{2}{c}{ CON } & \multicolumn{2}{l}{ Sprint } & \multicolumn{2}{l}{ Endurance } \\
& df & $\chi^{2}$ & df & $\chi^{2}$ & df & $\chi^{2}$ \\
\hline Soma & 30 & $166.08^{*}$ & 30 & 29.04 & 30 & $106.79^{*}$ \\
Nucleus & 14 & $133.02^{*}$ & 14 & 12.97 & 14 & $34.49^{*}$ \\
\hline
\end{tabular}

* Significant distribution differences at the 0.05 level. **All zeroweek animals were pooled into one distribution since there were no significant differences between groups at the start of the study $(\mathrm{n}=12)$. be attributed to the use of acute exercise programs of different types, intensities, and durations. Conversely, chronic programs of physical activity have consistently resulted in no changes in the volumes of the soma and nucleus ${ }^{9,10,11}$. These findings have been interpreted as reflecting an adaptation of the motor neuron to the new level of neuromuscular activity ${ }^{12}$. However, to date, all longitudinal studies have used an endurance swimming program of light to moderate intensity as the mode of exercise. It seemed logical to determine if these structural adaptations would be consistent using different intensities of training. Therefore, this investigation was designed to study the chronic effects of 2 well-defined interval running programs of different intensities on the soma and nuclear diameters of the working motor neurons.

Materials and methods. Normal, 72-day-old, male albino rats (Sprague-Dawley strain) were brought into the laboratory and assigned randomly to one of the following

1 This study was supported by National Institute of Health Grant HD 03918, Michigan State University, East Lansing, Michigan.

2 Acknowledgments. Gratitude is expressed to Barbara Wheaton for her technical assistance in the processing of tissues and R. E. Carrow for making his laboratory facilities available for this study.

3 Present address: Department of Biomechanics, College of Osteopathic Medicine, Michigan State University, East Lansing, Michigan 48824, USA.

4 J. E. Edstrom, J. comp. Neurol. 107, 295 (1957).

5 J. Konecki, Folia histochim. cytochim. 5, 79 (1967).

6 M. M. Aleksandrovskaie, Y. M. Geinisman, V. N. Larina and V. N. Mats., Bull. exp. Brain Med. 63, 103 (1967).

7 V. Y. Geinismann, V. M. Larina and V. M. Mats, Tsitologiya 12 $737(1970)$.

8 V. Y. Geinismann, V. M. Larina and V. M. Mats, Brain Res. 26 $247(1971)$

9 R. A. Kocher, J. comp. Neurol. 76, 341 (1916).

10 L. B. Gerchman, unpublished Ph. D. thesis, Department of Anatomy, Michigan State University, East Lansing, Michigan, 1968.

11 V. P. Tomanov and G. N. Krivitskaya, Bull. exp. Biol. Med. 63, 107 (1957)

12 R. E. Burke and V. R. Edgerton, Exercise Sci. Sports Rev. 3 31 (1975). 
activity groups: sedentary control (CON), sprint running (SPT) or endurance running (END). All animals were housed in individual sedentary cages and had access to food (Wayne Laboratory Blox) and water ad libitum. Treatments were initiated when the animals were 85 days of age and were continued for 12 weeks. The SPT and END animals were trained in a battery of individually controlled-running wheels ${ }^{13}$ on specific interval running programs conducted once daily, 5 days per week ${ }^{\mathbf{1 4}}$. 4 animals from each activity group were killed on the day that the activity treatments began. These animals were 85 days of age and were pooled into 1 group since statistical treatment of their data revealed no significant differences between the groups. This pooled group of animals is hereafter designated as control zero-week animals. 4 animals in each group were subsequently sacrificed at 169 days of age. Trained animals were killed $72 \mathrm{~h}$ after their last bout of exercise. All animals were sacrificed under anesthesia by an i.p. injection of $4 \mathrm{mg} / 100 \mathrm{~g} \mathrm{~b}$. wt of $6.48 \%$ sodium pentobarbital solution. Spinal cord segments L4 through S2 were removed, fixed in $10 \%$ buffered formalin for $24 \mathrm{~h}$ and embedded in paraffin. Serial cranial-caudal cross-sections, $7 \mu \mathrm{m}$ thick, were mounted on $35 \mathrm{~mm}$ leader film ${ }^{15}$ and stained with Luxol Fast Blue and counter-stained with Cresylecht Violet. For each animal, 60 motor neurons meeting the following criteria were used for analysis: a) each motor neuron had to possess a nucleolus and a distinct nuclear membrane; and b) each motor neuron had to be located in the somatic efferent (Lamina IX) portion of the right ventral horn ${ }^{16}$. Morphometric measurements were done using the technique described by Gilliam and Taylor ${ }^{17}$. Frequency distributions for the diameters of the soma and nucleus were analyzed using $\chi^{2}$ analyses of contingency tables (ACT). The 0.05 level of significance was established for all statistical analyses.
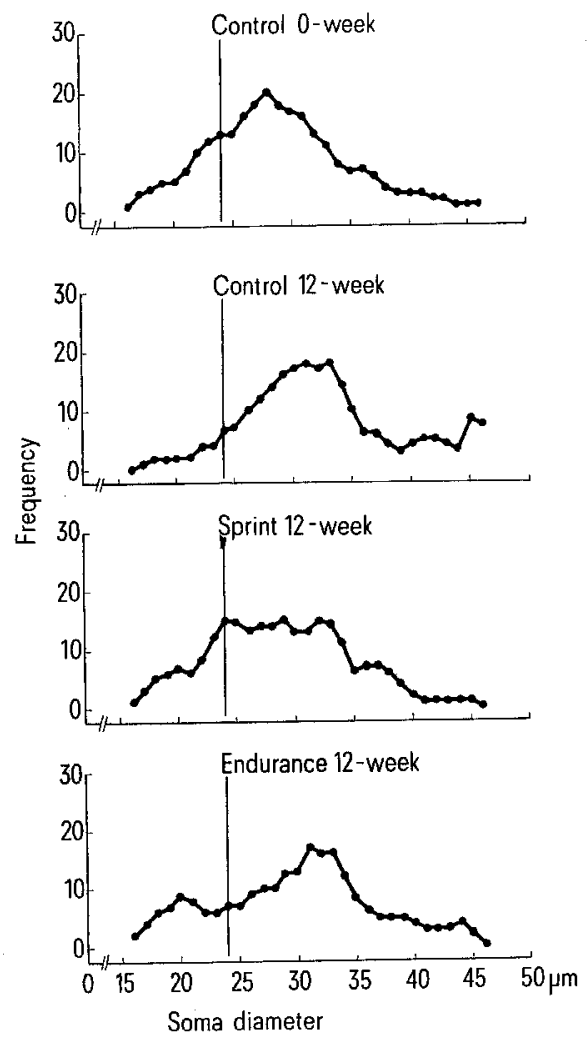

Fig. 1. Chronically induced changes in soma diameter after 12 weeks of interval training.
Results and discussion. Previous findings have indicated motor neurons return to 'normal' 18 activity $36-72 \mathrm{~h}$ following increased functional activity ${ }^{9}$. Since the animals in this study were sacrificed approximately $65-72 \mathrm{~h}$ after the last exercise period, we can assume that the motor neurons had recovered from the acute effects of the last exercise bout. The observed changes in the distributions of the morphological parameters reflected chronic changes specific to each exercise regimen. The results presented in table 1 show that the CON, SPT, and END frequency distribution for both soma and nucleus were significantly $(p<0.05)$ different from each other. Figures 1 and 2 depict these differences. Recently, Janzen typed alpha motor neurons in the rat according to size ${ }^{19}$. Using his data $( \pm 2 \mathrm{SD})$, the vertical lines shown in figure 1 differentiate between large $(>24 \mu \mathrm{m})$ and small $(<24 \mu \mathrm{m})$ alpha motor neurons. The vertical

13 R. L. Wells and W. W. Heusner, Lab. Anim. Bull. 21, 904 (1971).

14 R. C. Hickson, W. W. Heusner and W. D. Van Huss, J. appl. Physiol. 40, 868 (1976).

15 J. P. Pickett, W. B. Greene and J. R. Sommer, Archs Path. 77, 429 (1964).

16 Preliminary unpublished work was done to identify the location of the motor neurons innervating the plantar flexors of the hind limb. A small section of the right tibial nerve was removed proximal to the knee and spinal cord segments L4 to S1 inspected $4,7,10,14$ and 21 days post-operatively. Histological sections showed progressive chromatolysis of the motor neurons located in the somatic efferent portion of the ventral horn.

17 T. B. Gilliam and J. F. Taylor, Experientia, 32, 1219, (1976).

18 The stabilized intracellular environment following the acute effects of the increased functional activity.

19 R. W. C. Janzen, E.-J. Speckmann and H. Caspers, Cell Tiss. Res. 151,159 (1974).
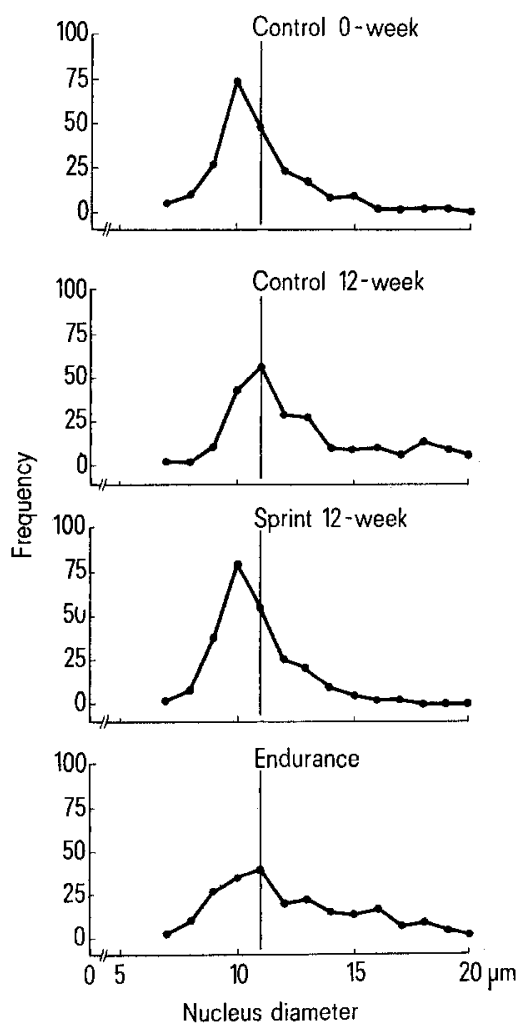

Fig. 2. Chronically induced changes in nuclei diameter after 12 weeks of interval training. 
lines drawn at the value 13 in figure 2 were arbitrarily established to act as a guideline for comparative purposes.

After 12 weeks of training, both the SPT and END groups demonstrated a 'retardation effect' in motor neuron size when compared to 12 -week control distribution (figures 1 and 2). Furthermore, the degree of 'retardation' is apparently dependent on the intensity of exercise used. This is shown in figures 1 and 2 with the SPT group demonstrating a greater number of smaller soma and nuclei than the END group. These findings are in contrast to earlier studies reporting no changes in motor neuron morphology between control and experimental groups following chronic activity $4,10,11$. It is the opinion of the authors that these observed differences are primarily due to 2 reasons: a) the training intensities used in earlier studies were not great enough to produce changes, and $b)$ the statistical treatment of the data may have masked any changes - any statistical analyses comparing the means of the groups in this study would have revealed no significant differences between the 3 groups. In fact, however, there are significant distribution differences as evidenced by the figures 1 and 2 .

A probable maturation effect can be observed between the control zero-week animals and the control 12-week animals (table 2). That is, the control 12-week distribution shows a greater number of larger motor neurons. In conclusion, it is evident that specific differences have been observed for the treatments used. It appears that motor neurons in a state of 'normality' reflect patterns of change which are specific to various chronic exercise regimens.

\title{
Suppression of delayed type hypersensitivity of mice by lead
}

\author{
S. Müller, K.-E. Gillert, Ch. Krause ${ }^{1}$, U. Gross ${ }^{2}$, J. L'Age-Stehr ${ }^{\mathbf{3}}$ and T. Diamantstein ${ }^{\mathbf{3}, 4}$ \\ Robert-Koch-Institut, Nordufer 20, D-1000 Berlin (West) 65, 27 September 1976
}

Summary. Application of lead suppressed the delayed type hypersensitivity (DTH) of mice induced by sheep red blood cells (SRBC). Inhibition of elicitation of DTH in primary as well as in secondary response was correlated with the concentration of lead in the blood of the mice.

It has been shown that several environmental contaminants ${ }^{5}$ including subclinical doses of lead $^{6}$ reduce the resistance of mice to bacterial infection. As reported recently, chronic exposure of mice to lead suppresses the humoral antibody response to sheep red blood cells (SRBC) as measured by the number of anti-SRBC producing spleen cells ${ }^{5}$. The authors concluded that the

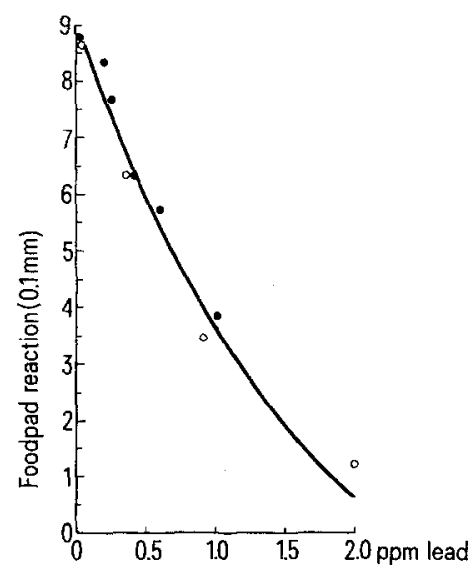

Fig. 1. Dose-dependent inhibition of DTH in mice exposed to lead. Levels of DTH elicited by inoculation of $10^{8} \mathrm{SRBC}$ into the right hind footpad of balb/c mice (6-8 weeks old) sensitized 4 days previously with $10^{5}$ SRBC i.v. DTH was measured $24 \mathrm{~h}$ after antigen challenge. Various amounts of lead acetate were injected i.p. in a volume of $0.5 \mathrm{ml}$ saline daily for 30 days. In the first experiment the mice received $0.25 \mathrm{mg}, 0.10 \mathrm{mg}$ and $0.025 \mathrm{mg}$ lead acetate daily $(0)$. In the second experiment the mice received $0.20 \mathrm{mg}, 0.10 \mathrm{mg}, 0.075 \mathrm{mg}$, $0.05 \mathrm{mg}$ and $0.025 \mathrm{mg}$ lead acetate daily $(\bullet)$. The lead content in the blood of mice was determined by means of an atomic absorption spectrophotometer immediately after DTH-measurement. Abscissa: ppm lead detected in whole blood. Ordinata: units of footpad reaction (1 unit $=0.1 \mathrm{~mm}$ ). Each point represents the mean value detected in each group of 10 mice. In the group of highest lead concentration, 4 mice died in consequence of lead intoxication. impaired resistance to the bacterial infection was probably due to a suppressed humoral antibody formation. But otherwise not only the humoral but also the cell mediated immunity (CMI), as well as nonspecific defense mechanisms due to phagocytic cells, are involved in these processes.

We investigated the effect of chronic lead exposure on the T-lymphocyte mediated and monocyte dependent delayed type hypersensitivity (DTH) against SRBC in mice. 10 mice in each group were injected i.p. once daily for 30 days with various amounts of lead acetate dissolved in saline. On the 30th day the mice were sensitized with $10^{5}$ SRBC i.v. To assess the DTH mice were challenged 4 days after sensitization by injection of $10^{8} \mathrm{SRBC}$ in $40 \mu 1$ of saline into the right hind footpad. As controls non-sensitized mice were injected into the footpad with the same dose of antigen. For this, lead-treated as well as non-treated animals were used. The footpad swelling was measured with a dial gauge caliper before antigen challenge, $24 \mathrm{~h}$, and $48 \mathrm{~h}$ after challenge according to Miller et al. ${ }^{7}$. The mice were killed and the lead content of the blood was determined by atomic absorption spectrophotometry.

1 Institut für Wasser-, Boden- und Lufthygiene, Corrensplatz 1, D-1000 Berlin (West) 33.

2 Institut für Pathologie, Klinikum Steglitz, Freie Universität Berlin, Hindenburgdamm 30, D-1000 Berlin (West) 45.

3 Immunologische Forschungseinheit, Klinikum Steglitz, Freie Universität Berlin, Hindenburgdamm 30, D-1000 Berlin (West) 45.

4 Acknowledgments. Supported by the 'Georg-und-Agnes-BIumenthal-Stiftung' and the 'Verein für Wasser-, Boden- und Lufthygiene'. We thank Miss Schachtschneider for the excellent technical assistance.

5 L. D. Koller and S. Kovacic, Nature $250,1.48$ (1974).

6 F. E. Hemphill, M. L. Kaeberle and W. B. Buck, Science 172, 1031 (1971).

7 T. E. Miller, G. B. Mackaness and P. H. LaGrange, J. Nat. Cancer Inst. 51, 1669 (1973). 